\author{
P. D. Home · S. J. Pocock • H. Beck-Nielsen · R. Gomis • \\ M. Hanefeld $\cdot$ H. Dargie $\cdot$ M. Komajda $\cdot$ J. Gubb $\cdot$ \\ N. Biswas $\cdot$ N. P. Jones
}

\title{
Rosiglitazone Evaluated for Cardiac Outcomes and Regulation of Glycaemia in Diabetes (RECORD): study design and protocol
}

Received: 25 November 2004 / Accepted: 28 April 2005 / Published online: 16 July 2005

(C) Springer-Verlag 2005

\begin{abstract}
Aims/hypothesis: Studies suggest that in addition to blood glucose concentrations, thiazolidinediones such as rosiglitazone improve some cardiovascular (CV) risk factors and surrogate markers, that are abnormal in type 2 diabetes. However, fluid retention might lead to cardiac failure in a minority of people. The aim of the Rosiglitazone Evaluated for Cardiac Outcomes and Regulation of Glycaemia in Diabetes (RECORD) study is to evaluate the long-term impact of these effects on $\mathrm{CV}$ outcomes, as well as on long-term glycaemic control, in people with type 2 diabetes. Materials and methods: RECORD is a 6-year, randomised, open-label study in type 2 diabetic patients with inadequate blood glucose control $\left(\mathrm{HbA}_{1} \mathrm{c} 7.1-9.0 \%\right)$ on metformin or sulphonylurea alone. The study is being performed in 327 centres in Europe and Australasia. After a 4-week run-in, participants were randomised by current treatment stratum to add-on rosiglitazone, metformin or sulphonylurea, with dose titration to a target $\mathrm{HbA}_{1} \mathrm{c}$ of $\leq 7.0 \%$. If confirmed
\end{abstract}

P. D. Home $(\bowtie)$

School of Clinical Medical Sciences-Diabetes,

University of Newcastle upon Tyne,

Medical School, Framlington Place,

Newcastle upon Tyne,

NE2 4HH, UK

e-mail: philip.home@newcastle.ac.uk

Tel.: +44-191-2227019

Fax: +44-191-2220723

P. D. Home

Newcastle Diabetes Centre,

Newcastle upon Tyne, UK

S. J. Pocock

London School of Hygiene and Tropical Medicine,

Medical Statistics Unit,

London, UK

H. Beck-Nielsen

Department of Endocrinology and Metabolism,

Odense University Hospital,

Odense, Denmark
$\mathrm{HbA}_{1} \mathrm{c}$ rises to $\geq 8.5 \%$, either a third glucose-lowering drug is added (rosiglitazone-treated group) or insulin is started (non-rosiglitazone group). The same criterion for failure of triple oral drug therapy in the rosiglitazone-treated group is used for starting insulin in this group. The primary endpoint is the time to first CV hospitalisation or death, blindly adjudicated by a central endpoints committee. The study aim is to evaluate non-inferiority of the rosiglitazone group vs the non-rosiglitazone group with respect to $\mathrm{CV}$ outcomes. Safety, tolerability and study conduct are monitored by an independent board. All CV endpoint and safety data are held and analysed by a clinical trials organisation, and are not available to the study investigators while data collection is open. Results: Over a 2-year period a total of 7,428 people were screened in 25 countries. Of these, 4,458 were randomised; 2,228 on background metformin, 2,230 on background sulphonylurea. Approximately half of the participants are male (52\%) and almost all are Caucasian (99\%). Conclusions/interpretation: The

R. Gomis

Endocrinology Department, Hospital Clinic,

Barcelona, Spain

M. Hanefeld

Centre for Clinical Studies, GWT Technical University,

Dresden, Germany

H. Dargie

Department of Cardiology, University of Glasgow,

Glasgow, Scotland, UK

M. Komajda

Cardiology Department, Pitié-Salpêtrière Hospital,

Paris, France

J. Gubb · N. P. Jones

GlaxoSmithKline Pharmaceuticals,

Harlow, Essex, UK

N. Biswas

GlaxoSmithKline Pharmaceuticals, Collegeville,

Philadelphia, PA, USA 
RECORD study should provide robust data on the extent to which rosiglitazone, in combination with metformin or sulphonylurea therapy, affects CV outcomes and progression of diabetes in the long term.

Keywords Cardiovascular outcomes - RECORD study · Rosiglitazone $\cdot$ Thiazolidinediones $\cdot$ Type 2 diabetes

Abbreviations AER: albumin excretion rate ALT: alanine aminotransferase $\cdot \mathrm{CV}$ : cardiovascular $\cdot \mathrm{CRP}$ : C-reactive protein - DSC-R: Diabetes Symptom Checklist revised version - DSMB: data safety monitoring board . FPG: fasting plasma glucose $\cdot \beta$ hCG: $\beta$-human chorionic gonadotrophin - hPI: intact human proinsulin - PAI-1: plasminogen activator inhibitor-1 - PPAR- $\gamma$ : peroxisome proliferator-activated receptor- $\gamma \cdot$ RECORD:

Rosiglitazone Evaluated for Cardiac Outcomes and Regulation of Glycaemia in Diabetes - UKPDS: UK Prospective Diabetes Study

\section{Introduction}

Type 2 diabetes is a progressive condition characterised by hyperglycaemia and other metabolic disorders, and their associated complications. Studies show that type 2 diabetes is significantly associated with all-cause and cardiovascular (CV) mortality [1], and that CV disease is the leading cause of death in people with type 2 diabetes [2].

Since insulin insensitivity has been identified as an important underlying or associated factor in the pathogenesis of type 2 diabetes and related CV disease [3, 4], drugs that ameliorate insulin insensitivity and the $\mathrm{CV}$ risk factors and markers associated with it, might be of benefit in reducing $\mathrm{CV}$ disease risk. This cluster of abnormalities includes insulin insensitivity itself, glucose intolerance, abdominal adiposity, dyslipidaemia (raised triglyceride and small, dense LDL particles, and decreased HDL cholesterol), raised blood pressure and microalbuminuria.

Rosiglitazone and pioglitazone are the two clinically available peroxisome proliferator-activated receptor- $\gamma$ (PPAR- $\gamma$ ) agonists, a class of oral glucose-lowering drugs that improves glycaemic control in type 2 diabetes by improving insulin sensitivity [5-11]. The Rosiglitazone Evaluated for Cardiac Outcomes and Regulation of Glycaemia in Diabetes (RECORD) study is concerned with rosiglitazone. Consistent with its insulin-sensitising effect, this drug has shown improvements in CV risk factors associated with insulin insensitivity, such as raised blood pressure, AER [12], plasminogen activator inhibitor-1 (PAI-1) [13] and C-reactive protein (CRP) [13, 14], and low serum HDL cholesterol (notably $\mathrm{HDL}_{2}$ ) $[8,15,16]$. Whilst increases in serum LDL cholesterol concentrations have been observed, these are associated with shifts towards larger, presumably less atherogenic LDL particles, making the significance of this observation unclear $[15,17]$.

An additional concern with this drug class, particularly the dual PPAR- $\alpha \gamma$ agonists that are under development, is fluid retention and, thus, whether this predisposes a pop- ulation already at risk of cardiac failure to a higher rate of possible adverse outcomes [18, 19]. Weight gain also occurs with these drugs, but this is consistent with improved glucose control, fat redistribution to less metabolically active peripheral adipose tissue, and some fluid retention [20]. The PPAR $-\gamma$ agonist troglitazone has been associated with rare cases of serious hepatic damage leading to liver failure, but there has been no evidence of this hepatotoxicity in wide clinical experience with rosiglitazone or pioglitazone $[21,22]$.

The glucose-lowering effects of oral glucose-lowering drugs were shown by the UK Prospective Diabetes Study (UKPDS) to translate into improved health outcomes [23]. A substudy of the UKPDS suggested that metformin had some additional advantage in cardiovascular risk protection [24]. Thiazolidinediones, as newer drugs, now need to be evaluated in the same way, which is the rationale for the current study.

In the management of type 2 diabetes, after lifestyle changes have been made in terms of diet (following dietary advice) and increased physical activity, drug therapy usually becomes necessary over time to maintain good glycaemic control. However, the ability of available drugs to maintain adequate long-term glycaemic control as a monotherapy is limited $[23,24]$, and the usual recourse is then to combination therapy with metformin plus a sulphonylurea. This dual therapy context appears to be the most logical in which to test the overall effectiveness of thiazolidinediones, although this complicates study design by requiring separate strata for those participants entering on metformin and those on a sulphonylurea. Furthermore, the expected continuing deterioration in glucose control during the course of the study leads to design issues over how to manage further intensification of treatment: should triple oral therapy or insulin be used? At which glycaemic thresholds should this occur?

This paper describes the RECORD study protocol, devised in an attempt to best meet these needs.

\section{Subjects and methods}

The study is being conducted according to Good Clinical (Research) Practice guidelines and the Declaration of Helsinki (1996) [25]. The conduct of each participating centre is monitored by regular visits from study staff. Two centres with suspected conduct problems were suspended from participation very early in the study. The study protocol was approved by ethics review committees or institutional review boards according to the local laws and customs of each participating country. Written informed consent was obtained from all participants before beginning protocol-specific procedures. Participants were informed of their right to withdraw from the study at any time. A steering committee meets to review progress every 6 months, and an independent data safety monitoring board (DSMB) has been established to monitor safety and outcomes throughout the course of the study (see below). The steering committee comprises six senior clinical ex- 
perts, one senior expert statistician and two sponsor employees with specialism in clinical research.

Participants In total, 7,428 people with type 2 diabetes were enrolled into the study, and 4,458 were randomised in 327 centres (secondary care clinics and general practitioner surgeries, including site management organisations and private diabetes clinics) in 25 countries in Europe and Australasia. Each centre attempted to enrol 10-20 people. Recruitment began in April 2001 and was completed in April 2003. People with type 2 diabetes were screened for eligibility for study entry according to the criteria presented in Table 1. The requirement for patients to be taking the maximum tolerated dose of their background monotherapy at study entry was to ensure compliance with the product licence for rosiglitazone at the time of study design.

Study design The study is a multicentre, randomised, openlabel, comparative, parallel-group trial. Eligible participants (see above) entered a 4-week run-in period that included reinforcement of lifestyle education (Fig. 1). During this period, subjects continued to take the oral glucoselowering drug (metformin or sulphonylurea) taken prior to entry into the study. At the end of the run-in period, randomisation is followed by planned participation for a minimum of 5 years (maximum of 21 visits) and a median of 6 years. After randomisation, participants continued their pre-study glucose-lowering therapy in addition to their study medication. An open-label design was dictated by the different timings of progression to insulin therapy in the rosiglitazone and comparator groups (see below), and by the considerable number of types and doses of the sulphonylurea medications used in the comparator arms.

Randomisation and treatments The treatment allocation schedule was computer generated in blocks and stratified according to background glucose-lowering medication (metformin or sulphonylurea). Participants were randomised centrally using an interactive voice response telephone system. If already taking a sulphonylurea, participants were randomised to either additional rosiglitazone or metformin. If already taking metformin, they were randomised to additional rosiglitazone or sulphonylurea (glyburide/ glibenclamide [normal or micronised], gliclazide or glimepiride; chosen according to local practice). It has been assumed that each of these three sulphonylureas is likely to have a similar effect on CV clinical outcomes. Each of these four treatment arms consists of approximately 1,100 people. Background glucose-lowering medications may be stopped or reduced at any time if intolerance develops or they become contraindicated (according to local practice).

Throughout the study, the protocol specifies that participants are to be treated using a target $\mathrm{HbA}_{1} \mathrm{c}$ of $\leq 7.0 \%$. If a person's $\mathrm{HbA}_{1} \mathrm{c}$ rises above $7.0 \%$ at any point after 8 weeks of treatment, the dose of the randomised study medication should be increased. If the initial dose of rosiglitazone $(4 \mathrm{mg})$ has been well tolerated, rosiglitazone may be increased to a maximum of $8 \mathrm{mg} /$ day (taken as 4 mg twice daily). Metformin, glibenclamide, gliclazide and

Table 1 Eligibility criteria for participation in RECORD study

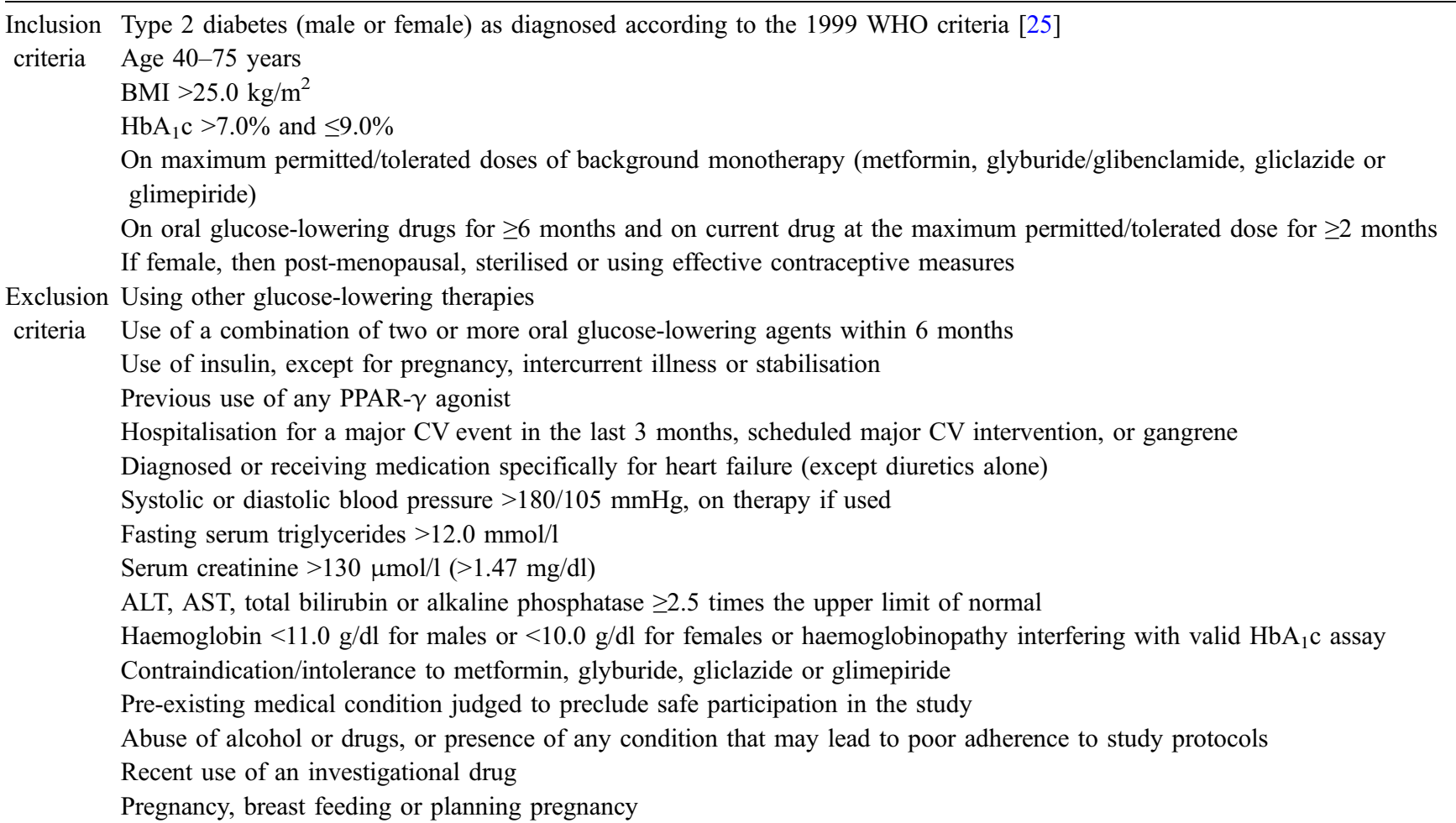




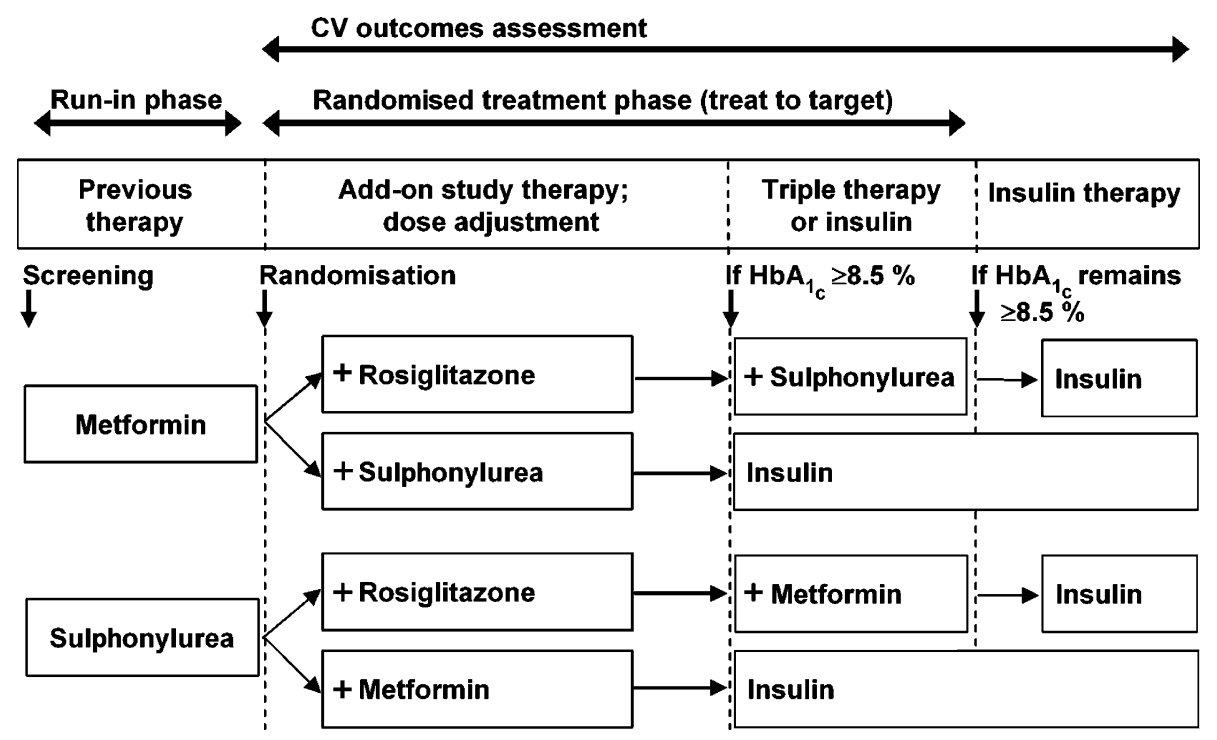

Fig. 1 A summary of the RECORD study design. After the run-in period, participants were randomised to add-on treatment with rosiglitazone or the oral glucose-lowering drug (metformin or sulphonylurea) not already being taken as background medication. If, during the study and despite titration (to a $\mathrm{HbA}_{1} \mathrm{c}$ of $7.0 \%$ ) of the study medication, $\mathrm{HbA}_{1} \mathrm{c}$ is $\geq 8.5 \%$ in two consecutive measure-

glimepiride may be similarly increased up to maximum dosages of 2,550, 15, 240 or $4 \mathrm{mg}$, respectively.

If a participant's $\mathrm{HbA}_{1} \mathrm{c}$ is $\geq 8.5 \%$ (confirmed by a second measurement at least 1 month later) despite having been on a maximum permitted or tolerated dose of add-on study medication for at least 8 weeks, additional therapy changes are made, as shown in Fig. 1. Participants in a rosiglitazone treatment arm have a third oral drug added, to make a triple oral combination treatment of rosiglitazone+sulphonylurea+metformin. Those on a metformin+ sulphonylurea combination start insulin with or without continuing metformin and/or sulphonylurea, according to local practice. If participants who are receiving triple therapy including rosiglitazone later have a confirmed $\mathrm{HbA}_{1} \mathrm{c} \geq$ $8.5 \%$, they start insulin therapy ( \pm metformin/sulphonylurea) and cease rosiglitazone therapy. This asymmetric approach to insulin therapy was judged the most appropriate design, being clinically rational without breaching contraindications to combination insulin+rosiglitazone use. Any participants starting insulin therapy, although withdrawn from the requirement to use their randomised treatment, continue to have CV outcomes monitored and evaluated as before until the planned end of the study. Blood glucose control and safety measurements and adverse events also continue to be collected. The $\mathrm{HbA}_{1} \mathrm{c}$ threshold for starting insulin or triple oral therapy was set at $8.5 \%$ to attempt to balance the need to avoid excessive hyperglycaemia against ensuring sufficient exposure to the randomised study medication.

Use of concomitant medications is permitted throughout the study, with the exception of glucose-lowering medications in participants taking the randomised treatments. ments $>1$ month apart (and at least 8 weeks from titration to maximum dose of study medication), participants using rosiglitazone will start a third oral glucose-lowering drug, while non-rosiglitazone users will transfer to insulin therapy. Insulin therapy will also be started in the rosiglitazone group if the same conditions are later met on triple therapy, with the rosiglitazone then being stopped

Visits and procedures Study visits are scheduled for the morning, after an overnight fast of $\geq 8 \mathrm{~h}$ and omission of any morning doses of glucose-lowering medications. Following screening (visit 1), a baseline (randomisation) visit (visit 2) was scheduled for 4 weeks later. While taking the randomised treatments, visits are scheduled to occur 2-monthly until month 12 , then 3-monthly to month 24 , then 4-monthly (all \pm 14 days). For those participants no longer taking randomised treatments, visits are scheduled to occur every 12 months, timed from the baseline visit.

During the screening visit (visit 1), the following procedures were performed after written informed consent was obtained: (1) completion of a checklist of inclusion/ exclusion criteria; (2) recording of clinical characteristics, including smoking and alcohol; (3) recording of medical history, including current signs/symptoms, glucose-lowering medications taken within the last 6 months, and other prescription-only medications taken within the last month; (4) physical examination, including standardised measurement of blood pressure, heart rate, height, body weight and WHR; (5) laboratory measurements of blood specimens (see below), including serum $\beta$-human chorionic gonadotrophin ( $\beta$ hCG) in premenopausal, non-sterilised women; and (6) standard 12-lead ECG. Lifestyle education was provided.

At the baseline visit (visit 2), vital signs and blood sampling were repeated, and the following procedures were completed: (1) first-pass morning urine sample for determination of the urinary albumin:creatinine ratio; (2) measurement of body weight; (3) the Diabetes Symptom Checklist, revised version (DSC-R) [26] and the Medical Care Utilization and Restricted Activity Days question- 
naires [27]; (4) a check for concomitant medications; (5) a check for any baseline signs/symptoms; and (6) a review of current diet and exercise.

Routine assessments made while patients are taking the randomised treatments include: (1) complete physical examination (yearly); (2) vital signs; (3) laboratory assessment of fasting blood specimens, including liver function monitoring; (4) first-pass morning urine sample for determination of the urinary albumin:creatinine ratio (months 6,12 , and then yearly); (5) the DSC-R (yearly); (6) the Medical Care Utilization and Restricted Activity Days questionnaires; (7) body weight; (8) smoking habits; (9) WHR; (10) standard 12-lead ECG (yearly); (11) recording of concomitant medications; (12) recording of adverse experiences; and (13) a review of current diet and exercise. Add-on study medication was dispensed following the completion of all assessments.

For those participants withdrawn from randomised treatments (see above), the following assessments are made yearly: (1) vital signs; (2) non-fasting blood specimens $\left(\mathrm{HbA}_{1} \mathrm{c}\right.$ and liver function tests only); (3) body weight; (4) DSC-R; (5) the Medical Care Utilization and Restricted Activity Days questionnaires; and (6) complete physical examination. Contact is made between these visits by telephone, following the same frequency as when on randomised treatments, for completion of the Medical Care Utilization and Restricted Activity Days questionnaires and ascertainment of cardiovascular outcomes.

Methods of evaluation A central laboratory is being used for all routine laboratory assessments (Quest Diagnostics, Heston, UK). Assessment of fasting blood specimens includes evaluation of $\mathrm{HbA}_{1} \mathrm{c}$ (all visits), plasma glucose (all visits), insulin and proinsulin (baseline and 12, 18, 24 months, then yearly), standard lipid profile (baseline and $12,18,24$ months, then yearly) and surrogate markers (PAI1 antigen, apolipoprotein B, CRP and fibrinogen; baseline and 12, 24, 36 and 60 months). Other laboratory assessments include a urinary albumin:creatinine ratio, as well as safety assessments, including standard biochemistry and haematology markers, pregnancy screen, hepatitis testing and liver function tests.

$\mathrm{HbA}_{1} \mathrm{c}$ is measured by HPLC using a DCCT-harmonised Biorad Variant $\mathrm{HbA}_{1} \mathrm{c}$ assay (Hercules, CA, USA). Fasting plasma glucose concentration is measured using an enzymatic method and is read biochromatically. Serum immunoreactivity is determined by a two-site fluoroimmunometric assay (Perkin Elmer, Turku, Finland). The assay is specific for insulin, with negligible cross-reactivity for proinsulin and its intermediates (intact human proinsulin [hPI] 0.1\%; des 32,33 hPI 0.4\%; des 64,65 hPI $66 \%$ ). Intact proinsulin is measured using a two-site fluoroimmunometric assay. The assay typically shows $<1 \%$ cross-reaction with insulin and 32,33 split proinsulin at concentrations of 2,500 and $400 \mathrm{pmol} / \mathrm{l}$, respectively. There is no detectable cross-reaction with C-peptide. Urinary albumin is measured by fixed-time nephelometry. Urinary creatinine is measured by a kinetic modification of the Jaffé method in which creatinine reacts with picric acid at alkaline $\mathrm{pH}$ to form a yellow-orange complex. PAI-1 antigen is quantified using a Biopool TintElize enzyme immunoassay kit (Ventura, CA, USA). Apolipoprotein B and CRP are measured by fixed-time nephelometry. Fibrinogen is measured using photo-optical clot detection in plasma when thrombin is added. The chemiluminometric BhCG immunoassay (Bayer Advia Centaur, Walpole, MA, USA) is used to measure $\beta$ hCG.

Health status and pharmacoeconomic outcomes are assessed using the DSC-R [26] and the Medical Care Utilization and Restricted Activity Days questionnaires [27]. The DSC-R is a 34-item self-report questionnaire comprising eight subdimensions (hyperglycaemic, hypoglycaemic, neurological-pain, neurological-sensory, cardiovascular, psychological-cognitive, psychological-fatigue and ophthalmological). Each of the 34 items is scored on a dichotomous scale for the presence/absence of the symptom. If a symptom is present, the degree of discomfort is recorded on a five-point Likert scale. Medical care utilisation data, including hospitalisations, accident and emergency unit visits, and non-protocol visits to or by any doctor, are collected in the trial as events requiring the intervention of a health-care professional. In addition, days of restricted activity (during which the participant is unable to perform the normal activities of daily life) over the 7 days prior to the scheduled visit are recorded. For Medical Care Utilization and Restricted Activity Days assessments, the relationship to diabetes (direct, indirect or none) is also recorded.

The DSC-R will be analysed according to the developer's instructions. Pharmacoeconomic outcomes include the frequency of casualty department visits, number of days hospitalised, unscheduled visits to study investigators, and restricted activity days.

Primary efficacy outcome The primary efficacy variable is the combined endpoint of $\mathrm{CV}$ death or CV hospitalisation. Its primary analysis will be time to first $\mathrm{CV}$ hospitalisation or $\mathrm{CV}$ death. The $\mathrm{CV}$ death component will include death following heart failure or acute myocardial infarction, sudden death, and death caused by acute vascular events. Cardiovascular hospitalisation will include hospitalisation due to acute myocardial infarction, CHF, stroke, unstable angina pectoris, transient ischaemic attack, unplanned invasive $\mathrm{CV}$ therapeutic procedure, amputation of extremities, or for a definite CV reason not defined by the protocol. A clinical endpoints committee (CEC) with vascular expertise has been appointed to review and adjucate all potential CV hospitalisation and CV death endpoints. This committee is blind to study medication and independent of the steering committee and study sponsor. For the sponsor to comply with its legal obligations to regulatory authorities, endpoints that meet the definition of 'serious adverse events' are reported to its clinical safety department. This department is separated by an information firewall from the sponsor's staff who work on clinical trials. 
Secondary efficacy outcomes Secondary measures of CV and diabetes-related endpoints are: (1) all-cause mortality; (2) definite heart failure; and (3) microvascular endpoints and combined $\mathrm{CV}$ hospitalization or $\mathrm{CV}$ death endpoint plus microvascular endpoints. Microvascular outcomes are assessed from diabetes-related (as determined by a local investigator) adverse events only, including foot ulceration, progression to dialysis, laser photocoagulation and blindness. Secondary endpoints will be analysed both in terms of time to first event and frequency of events.

Assessment of cardiac failure is very variable between clinicians; this poses a problem when studying drugs that cause fluid retention. Accordingly, hospitalisation (as for most other $\mathrm{CV}$ endpoints) and confirmation on review by the clinical endpoints committee are required for this to be included as an endpoint.

The following were defined as secondary variables for the metabolic measures: (1) changes from baseline in $\mathrm{HbA}_{1} \mathrm{c}$ and fasting plasma glucose (FPG) and the proportion of participants achieving predefined targets of glycaemic control (FPG $\leq 7.0 \mathrm{mmol} / \mathrm{l}$ and $\mathrm{HbA}_{1 \mathrm{c}} \leq 7.0 \%$ ); (2) failure of glycaemic control (addition of a third oral glucose-lowering agent or initiation of insulin treatment); (3) serum insulin and proinsulin; (4) urinary albumin:creatinine ratio; (5) insulin sensitivity and islet beta-cell function, as estimated by homeostasis model assessment [28]; (6) serum lipids; (7) NEFA; (8) PAI-1 antigen; (9) fibrinogen; (10) apolipoprotein B; and (11) CRP.

Safety assessments Changes in findings on physical examination (including body weight), vital signs, clinical laboratory tests (including liver function tests) and ECGs, and abnormal experiences are assessed throughout the course of the study by the DSMB. If alanine aminotransferase (ALT) is more than three times the upper limit of the normal range, or the participant reports symptoms suggestive of hepatic dysfunction (unexplained nausea, vomiting, abdominal pain, fatigue, anorexia and/or dark urine) at any visit after randomisation, evaluation for viral hepatitis (A, B and $\mathrm{C}$ ) is performed and liver function tests are assessed within 1 week. If ALT remains more than three times the upper limit of the normal range, the individual is withdrawn from randomised treatment and transferred to monitoring of $\mathrm{CV}$ outcomes and glycaemic control only (see above) for the planned duration of the study.

The DSMB meets independently of the steering committee, and is separated from staff of the monitoring organisation and sponsor by an information firewall. Stringent statistical criteria have been set prospectively for early study termination in the event of a clear-cut difference between the treatment groups with respect to all-cause mortality. A stopping guideline is being applied to interim analyses for overwhelming evidence of benefit/harm using the Haybittle-Peto procedure [29] with a fixed nominal $p$ value of 0.0005 (one-sided) for the comparison of the combined rosiglitazone group with the combined metformin+sulphonylurea group. For evidence of harm of the combined rosiglitazone group, the criterion is evaluated annually. For evidence of benefit, the criterion is evaluated after the end of year 3 of the study and annually thereafter. In addition, the DSMB may also recommend termination of the study for other serious safety reasons. A protocol has been established to ensure the rapid consideration and execution of such a recommendation throughout the geographical reach of the study.

Statistical analysis A sample size of 4,000 participants followed for a median of 6 years (assuming a 2-year recruitment period) was estimated to be sufficient for the primary objective of this study: to compare the time to the combined $\mathrm{CV}$ endpoint (CV death or $\mathrm{CV}$ hospitalisation) between those participants randomised to add-on treatment with rosiglitazone and those randomised to add-on treatment with sulphonylurea, pooling the data across study treatment arms. RECORD was designed as a non-inferiority trial. The rosiglitazone group is defined to be noninferior to the non-rosiglitazone control group if the upper limit of the $95 \%$ confidence interval for the hazard ratio falls below 1.20. A total of 2,000 participants per treatment stratum was estimated to give $99.2 \%$ power to confirm non-inferiority when the control group has an $11 \%$ event rate per year $3 \% \mathrm{CV}$ deaths and $8 \% \mathrm{CV}$ hospitalisations) $[30,31]$ and $2 \%$ of participants are lost to follow-up each year.

For the primary $\mathrm{CV}$ endpoint, the times to event will be summarised using Kaplan-Meier survival curves and compared between treatment groups using the proportional hazards model, with background therapy (metformin or sulphonylurea) as a covariate. A sensitivity analysis will be performed to assess the influence of geographical region on the conclusions. All supporting endpoints will test the null hypothesis of no treatment difference, using two-sided tests at the 95\% significance level. Participants who do not achieve the endpoint during their time in the study will have their data censored based on the date of final observed contact.

For metabolic endpoints $\left(\mathrm{HbA}_{1} \mathrm{c}, \mathrm{FPG}\right.$, insulin, insulin sensitivity, islet beta-cell function and lipids) treatment differences will be assessed at 3 years and study end using analysis of covariance adjusted for the values of the measure at baseline and screening. Additionally, a subgroup analysis of the metabolic endpoints of the first 1,040 or more participants to reach 18 months of therapy was planned prospectively, and has been completed [32].

For blood pressure a 6-month substudy (and extension) using ambulatory devices in a limited number of centres has been completed [33]. For the DSC-R, at each visit, the item scores for each participant will be averaged for each subdimension and for the overall 34 items, and the changes from baseline will be calculated. For the overall mean score, treatment differences over time will be assessed using a multivariate linear model analysis. Assessment of pharmacoeconomic endpoints at study end will use a Poisson regression model to estimate the event rate per 1,000 subject-days.

For efficacy analyses, the primary analysis population was defined as those participants who were randomised and received at least one dose of add-on study medication 
(the intention-to-treat population). Efforts are being made to collect $\mathrm{CV}$ endpoint data to study termination even in those withdrawing from the study, such as those who move away from their study centre. A secondary population, for the primary CV endpoint only, will be the 'per protocol' population, defined as participants who were randomised and still on the randomly allocated treatment up to 30 days before the primary CV endpoint or the time of non-inclusion. For a non-inferiority study, a per protocol analysis carries potentially less bias in favour of the tested drug than an intention-to-treat analysis. All individuals who receive at least one dose of randomised study medication will be assessed for clinical safety and tolerability.

Data analysis is provided by the study's clinical trial contract organisation (Quintiles, Bracknell, UK). Confirmatory analyses of the data will be conducted within the institution to which the independent statistician member of the steering committee (S. Pocock) is affiliated. Data are not made available to the sponsor except as planned analyses or descriptions approved by the steering committee.

\section{Results}

Screening for the study began on 23 April 2001 and the last individual was randomised on 29 April 2003. During this period 7,428 individuals were screened in 25 countries. Of these, 4,458 were randomised; 2,228 on background metformin, 2,230 on background sulphonylurea. The numbers randomised in each country were as follows: Australia 51, Belgium 104, Bulgaria 204, Croatia 274, Czech Republic 145, Denmark 57, Estonia 220, Finland 193, France 86, Germany 178 , Greece 139, Hungary 400, Italy 116, Latvia 173, Lithuania 134, the Netherlands 76, New Zealand 36, Poland 363, Romania 157, Russia 149, Slovakia 325, Spain 64, Sweden 469, Ukraine 103, UK 242.

Demographic details, baseline assessments and clinical history are summarised in Table 2. Approximately half of the participants are male (52\%) and almost all $(99 \%)$ are Caucasian. Patients have a mean age of 58 years and time since diagnosis of type 2 diabetes is 7 years. Compared with those in the background sulphonylurea stratum, the participants in the background metformin stratum are younger and more obese, with a shorter time since diagnosis (Table 2). They otherwise appear similar.

\section{Discussion}

While preliminary data concerning the impact of thiazolidinediones on insulin insensitivity and its associated cluster of CV risk factors appear promising [5-17], there is a need to investigate whether these effects will translate into improved CV outcomes and to assess the long-term efficacy and safety profile of this class of drugs. Such a
Table 2 Baseline characteristics of the people with type 2 diabetes who were randomised, divided according to background therapy

Data are means \pm SD, or $n(\%)$

\begin{tabular}{|c|c|c|c|}
\hline & Background metformin & Background sulphonylurea & All \\
\hline$N$ & 2,228 & 2,230 & 4,458 \\
\hline \multicolumn{4}{|l|}{ Demographic details } \\
\hline Age (years) & $57.1 \pm 8.1$ & $59.7 \pm 8.3$ & $58.4 \pm 8.3$ \\
\hline Men & $1,187(53)$ & $1,111(50)$ & $2,298(52)$ \\
\hline Caucasian & 2,198 (99) & 2,212 (99) & 4,410 (99) \\
\hline Duration from diagnosis (years) & $6.3(4.5)$ & $7.9(5.5)$ & $7.1(5.1)$ \\
\hline \multicolumn{4}{|l|}{ Baseline measurements } \\
\hline Weight (kg) & $93.5 \pm 16.5$ & $84.8 \pm 14.4$ & $89.2 \pm 16.1$ \\
\hline BMI $\left(\mathrm{kg} / \mathrm{m}^{2}\right)$ & $32.8 \pm 5.1$ & $30.3 \pm 4.1$ & $31.5 \pm 4.8$ \\
\hline Waist circumference $(\mathrm{cm})$ & $107.8 \pm 11.8$ & $101.7 \pm 11.0$ & $104.8 \pm 11.8$ \\
\hline Systolic blood pressure (mmHg) & $139.4 \pm 16.0$ & $138.2 \pm 14.9$ & $138.8 \pm 15.4$ \\
\hline Diastolic blood pressure $(\mathrm{mmHg})$ & $83.7 \pm 8.6$ & $82.3 \pm 8.0$ & $83.0 \pm 8.3$ \\
\hline $\mathrm{HbA}_{1} \mathrm{c}(\%)$ & $7.82 \pm 0.66$ & $7.97 \pm 0.73$ & $7.90 \pm 0.70$ \\
\hline LDL cholesterol (mmol/l) & $3.18 \pm 0.90$ & $3.39 \pm 0.91$ & $3.29 \pm 0.91$ \\
\hline HDL cholesterol (mmol/l) & $1.20 \pm 0.29$ & $1.20 \pm 0.30$ & $1.20 \pm 0.29$ \\
\hline Triglycerides $(\mathrm{mmol} / \mathrm{l})$ & $2.32 \pm 1.39$ & $2.25 \pm 1.67$ & $2.28 \pm 1.54$ \\
\hline \multicolumn{4}{|l|}{ Other clinical history } \\
\hline Ischaemic heart disease & $316(14)$ & 418 (19) & $734(16)$ \\
\hline Cerebrovascular disease & $92(4)$ & $103(5)$ & $195(4)$ \\
\hline Peripheral arterial disease & $91(4)$ & $166(7)$ & $257(6)$ \\
\hline Retinopathy & $182(8)$ & $328(15)$ & $510(11)$ \\
\hline Neuropathy & $107(5)$ & $165(7)$ & $272(6)$ \\
\hline Diagnosed hypertension & $1,476(66)$ & $1,447(65)$ & $2,923(66)$ \\
\hline Diagnosed lipid disorder & $830(37)$ & $677(30)$ & $1,521(34)$ \\
\hline Current smoker & $394(18)$ & $314(14)$ & $708(16)$ \\
\hline Former smoker & $642(29)$ & $465(21)$ & $1,107(25)$ \\
\hline
\end{tabular}


study will also address concerns over cardiac failure; confirm that the better health outcomes associated with improved glucose control, as reported by the UKPDS [23], are applicable to this group of drugs; and allay concerns based on LDL cholesterol concentrations rather than LDL particle atherogenicity. In the chosen context of people with diabetes initially inadequately controlled on metformin or sulphonylurea medication, and with comparison of rosiglitazone addition to these therapies with the standard combination of metformin and sulphonylurea over a period of up to 5-7 years, the RECORD study should provide valuable information, both on $\mathrm{CV}$ outcomes and long-term glycaemic control in individuals with limited treatment options other than the addition of insulin therapy.

As a result of necessary compromises of study design, the RECORD study protocol has a number of strengths and weaknesses. Although the frequency of adverse CV events is high in people with type 2 diabetes - as high as in a non-diabetic population with declared vascular disease [34] — with the assumed event rate and specified limit of non-inferiority margin, the study was designed to follow at least 4,000 people for an average of 6 years. Furthermore, the context of the current study is rosiglitazone in combination with standard oral glucose-lowering therapies, while current European International Diabetes Federation (IDF) guidelines suggest limits to the extent of hyperglycaemia that is acceptable before insulin therapy is introduced [35]. Together, these criteria limit the patient population that can be recruited into the study, implying the need for the participation of a large number of centres, each recruiting a relatively small number of participants. However, this does have the benefit of making the results more widely generalisable across the European population. In addition, a composite $\mathrm{CV}$ endpoint is needed to provide adequate statistical power, since the use of other preventative drug therapy (such as aspirin, ACE inhibitors and statins), which cannot of course be denied to participants, is likely to reduce the background event rate below that estimated from earlier studies.

Conducting a large multicentre study of this duration, involving two comparator drug types, the need for titration of therapies, a narrow glucose control window, and the need to capture diverse outcomes, presents its own problems in terms of recruitment, protocol compliance and subject retention. This has been addressed by substantial external monitoring of study centres to optimise protocol adherence. Investigator continuity is also an issue; this is achieved through repeated national and regional meetings and newsletters, as well as frequent contact with monitoring staff. Specific programmes address participant retention issues.

The asymmetry of the study design in terms of the initiation of insulin therapy stems from the need to address the continued deterioration of blood glucose control (resulting from the progressive loss of islet beta-cell function $[23,24])$. The study needs to ensure significant exposure to the trial medications in one group, while avoiding pe- riods of more marked hyperglycaemia arising from the absence of other oral glucose-lowering drugs of similar efficacy in the other group. In one sense this asymmetry may not matter, as the earlier (in time not glucose control) use of insulin in the non-rosiglitazone groups is clinically pragmatic and allows the putative advantages and disadvantages of rosiglitazone to continue to be compared with standard glucose-lowering medications. On the other hand, it may also bias the study against rosiglitazone if the glucose-lowering properties of insulin are used aggressively.

RECORD is an open-label study, reflecting the difficulties of providing dummy versions of the five oral add-on treatments at different doses, and of persuading participants who are often already using a variety of other therapies to take both active and dummy treatments over long periods. In any case, the study could not proceed in a blinded fashion at the time of introduction of insulin therapy, which differs in the rosiglitazone and non-rosiglitazone arms. Open-label studies carry a risk of differential assessments of outcomes because of the differential expectations of the participants and investigators. The use of hard primary endpoints (death and hospitalisation) assessed by a blinded endpoints group, together with biochemical measurements in a central laboratory, should reduce this risk, as should the tight external monitoring by a contract clinical trial organisation (Quintiles), appointed by the sponsor.

While the primary endpoint is well defined, the diversity of macrovascular and microvascular assessments and the needs of safety monitoring for a drug of a novel class all lead to a plethora of secondary endpoints, which may increase the risk of statistically significant chance findings, but may also allow for the cross-checking of consistency between measures which on a priori grounds may be associated. The deployment of a steering committee and an independent Data Safety Monitoring Board, both comprising senior clinical experts and external statisticians as full members, should help ensure the availability of appropriate expertise in trial design, execution, interim monitoring, analysis and reporting.

The study has recruited 2,160 women (48\%). This can be considered a strength of the study, since women are often not proportionately represented in CV outcome trials or in intervention studies assessing the treatment of type 2 diabetes.

Long-term clinical outcome trials such as RECORD provide the opportunity to test the safety and tolerability of newer agents in relation to standard therapies; in this study, safety data for rosiglitazone in combination therapy is anticipated to comprise more than 10,000 person-years of experience. Current experience with rosiglitazone suggests that it lacks a troglitazone-like hepatotoxicity $[21,36]$, but liver function tests will, nevertheless, be extensively monitored. Equally, the issue of whether weight gain or fluid retention associated with thiazolidinedione therapy has any long-term cardiac sequelae should be resolved by the RECORD study. 
In conclusion, results from this study will provide a clearer picture of the benefits of rosiglitazone therapy vs traditional agents with more established efficacy and safety profiles. In particular, it will investigate the premise that thiazolidinediones, which improve glycaemic control by decreasing insulin insensitivity, reduce the incidence of macrovascular complications in individuals with type 2 diabetes.

Acknowledgements The investigators are grateful to the people with type 2 diabetes participating in this study for their time and commitment.

\section{Duality of interest}

The RECORD study sponsor is GlaxoSmithKline, the manufacturer of rosiglitazone. Members of the steering committee, the DSMB and the Clinical Endpoints Committee, or their institutions, are remunerated for time and expenses and, in some cases, other activities relating to the sponsor. J. Gubb, N. Biswas and N. P. Jones are employees of GlaxoSmithKline. Local investigators and/or their institutions are paid fees per participant for study activities; some will also be remunerated for other activities relating to the sponsor.

\section{References}

1. Wei M, Gaskill SP, Haffner SM, Stern MP (1998) Effects of diabetes and level of glycemia on all-cause and cardiovascular mortality. The San Antonio Heart Study. Diabetes Care 21: 771167-1172

2. Morrish NJ, Wang S-L, Stevens LK, Fuller JH, Keen H and the WHO Multinational Study Group (2001) Mortality and causes of death in the WHO Multinational Study of Vascular Disease in Diabetes. Diabetologia 44(Suppl 2):S14-S21

3. Reaven GM (1988) Banting lecture: role of insulin resistance in human disease. Diabetes 37:1595-1607

4. Haffner SM, Valdez RA, Hazuda HP et al (1992) Prospective analysis of the insulin-resistance syndrome (syndrome $\mathrm{X}$ ). Diabetes 41:715-722

5. Mayerson AB, Hundal RS, Dufour S et al (2002) The effects of rosiglitazone on insulin sensitivity, lipolysis, and hepatic and skeletal muscle triglyceride content in patients with Type 2 diabetes. Diabetes 51:797-802

6. Miyazaki Y, Glass L, Triplitt C et al (2001) Effect of rosiglitazone on glucose and non-esterified fatty acid metabolism in Type II diabetic patients. Diabetologia 44:2210-2219

7. Carey DG, Cowin GJ, Galloway GJ et al (2002) Effect of rosiglitazone on insulin sensitivity and body composition in type 2 diabetic patients. Obes Res 10:1008-1015

8. Lebovitz HE, Dole JF, Patwardhan R, Rappaport EB, Freed MI (2001) Rosiglitazone monotherapy is effective in patients with Type 2 diabetes. J Clin Endocrinol Metab 86:280-288

9. Hällsten K, Virtanen KA, Lönnqvist F et al (2002) Rosiglitazone but not metformin enhances insulin- and exercise-stimulated skeletal muscle glucose uptake in patients with newly diagnosed type 2 diabetes. Diabetes 51:3479-3485

10. Aronoff S, Rosenblatt S, Braithwaite S et al (2000) Pioglitazone hydrochloride monotherapy improves glycemic control in the treatment of patients with type 2 diabetes. Diabetes Care 23: 1605-1611

11. Miyazaki Y, Mahankali A, Matsuda M et al (2001) Improved glycemic control and enhanced insulin sensitivity in type 2 diabetic subject treated with pioglitazone. Diabetes Care 24: 710-719

12. Bakris G, Viberti G, Weston WM, Heise M, Porter LE, Freed MI (2003) Rosiglitazone reduces urinary albumin excretion in type II diabetes. J Hum Hypertens 17:7-12
13. Raji A, Seely EW, Bekins SA, Williams GH, Simonson DC (2003) Rosiglitazone improves insulin sensitivity and lowers blood pressure in hypertensive patients. Diabetes Care 26:172178

14. Haffner SM, Greenberg AS, Weston WM, Chen H, Williams K, Freed MI (2002) Effect of rosiglitazone treatment on nontraditional markers of cardiovascular disease in patients with Type 2 diabetes mellitus. Circulation 106:679-684

15. Freed MI, Ratner R, Marcovina SM et al (2002) Effects of rosiglitazone alone and in combination with atorvastatin on the metabolic abnormalities in Type 2 diabetes mellitus. Am J Cardiol 90:947-952

16. Ovalle F, Bell DS (2002) Effect of thiazolidinediones on highdensity lipoprotein subfractions. Endocr Pract 8:102-104

17. Cheng-Lai A, Levine A (2000) Rosiglitazone: an agent from the thiazolidinedione class for the treatment of Type 2 diabetes. Heart Dis 2:326-333

18. Nesto RW, Bell D, Bonow RO et al (2003) Thiazolidinedione use, fluid retention, and congestive heart failure. A consensus statement from the American Heart Association and American Diabetes Association. Circulation 108:2941-2948

19. Tang WHW, Francis GS, Hoogwerf BH, Young JB (2003) Fluid retention after initiation of thiazolidinedione therapy in diabetic patients with established chronic heart failure. J Am Coll Cardiol 41:1394-1398

20. King $A B$ (2000) A comparison in a clinical setting of the efficacy and side effects of three thiazolidinediones. Diabetes Care 23:557

21. Lebovitz HE, Kreider M, Freed MI (2002) Evaluation of liver function in type 2 diabetic patients during clinical trials: evidence that rosiglitazone does not cause hepatic dysfunction. Diabetes Care 25:815-821

22. Chilcott J, Tappenden P, Jones ML, Wight JP (2001) A systematic review of the clinical effectiveness of pioglitazone treatment of type 2 diabetes mellitus. Clin Ther 23:1792-1823

23. UK Prospective Diabetes Study (UKPDS) Group (1998) Intensive blood-glucose control with sulphonylureas or insulin compared with conventional treatment and risk of complications in patients with type 2 diabetes (UKPDS 33). Lancet 352:837-853

24. UK Prospective Diabetes Study (UKPDS) Group (1998) Effect of intensive blood-glucose control with metformin on complications in overweight patients with Type 2 diabetes (UKPDS 34). Lancet 352:854-865

25. World Medical Association (1997) Declaration of Helsinki. Recommendations guiding physicians in biomedical research involving human subjects. JAMA 277:925-926

26. Grootenhuis PA, Snoek FJ, Heine RJ, Bouter LM (1994) Development of a Type 2 diabetes symptom checklist: a measure of symptom severity. Diabet Med 11:253-261

27. Drummond MF, O'Brien B, Stoddart GL, Torrance GW (eds) (1997) Methods for the Economic Evaluation of Health Care Programmes, 2nd edn. Oxford University Press, New York

28. Matthews DR, Hosker JP, Rudenski AS, Naylor BA, Treacher DF, Turner RC (1985) Homeostasis model assessment: insulin resistance and $\beta$-cell function from fasting plasma glucose and insulin concentrations in man. Diabetologia 28:412-419

29. Jennison C, Turnbull BW (2000) Group Sequential Methods with Application to Clinical Trials, Chapman and Hall/CRC, Boca Raton (Section 2.7.2)

30. Goldberg RB, Mellies MJ, Sacks FM et al (1998) Cardiovascular events and their reduction with pravastatin in diabetic and glucose-intolerant myocardial infarction survivors with average cholesterol levels: subgroup analyses in the Cholesterol And Recurrent Events (CARE) trial. Circulation 98:2513-2519

31. Heart Outcomes Prevention Evaluation (HOPE) Study Investigators (2000) Effects of ramipril on cardiovascular and microvascular outcomes in people with diabetes mellitus: results of the HOPE study and MICRO-HOPE substudy. Lancet 355: 253-259 
32. Home PD, Pocock S, Beck-Nielsen H et al (2004) Rosiglitazone evaluated for cardiac outcomes and regulation of glycaemia in diabetes (RECORD): an interim analysis of glycaemia at 18 months. Diabetologia 47(Suppl 1):A262 (Abstract no. 725)

33. Oshinyemi K, Garcia S, Curtis P, Zambanini A, Stewart M (2004) Evaluation of the effects of rosiglitazone combination therapy on ambulatory blood pressure after 6 months; a 12 month sub-study of the RECORD trial in people with Type 2 diabetes mellitus. Diabetologia 47(Suppl 1):A262 (Abstract no. 724)
34. Haffner SM, Lehto S, Rönnemaa T, Pyörälä K, Laakso M (1998) Mortality from coronary heart disease in subjects with Type 2 diabetes and in nondiabetic subjects with and without prior myocardial infarction. N Engl J Med 339:229-234

35. European Diabetes Policy Group 1999 (1999) A desktop guide to Type 2 diabetes mellitus. Diabet Med 16:716-730

36. Sheen AJ (2001) Hepatotoxicity with thiazolidinediones: is it a class effect? Drug Saf 24:873-888 